\title{
THE TRANSIENT SEPARATION OF MULTICOMPONENT MIXTURES IN A CYLINDRICAL THERMOGRAVITATIONAL COLUMN
}

\author{
Sofia V. Kozlova ${ }^{1 *}$ and Ilya I. Ryzhkov ${ }^{1,2}$ \\ ${ }^{1}$ Institute of Computational Modelling $S B R A S$, \\ 50/44 Akademgorodok, 660036, Krasnoyarsk, Russia \\ ${ }^{2}$ Siberian Federal University \\ 79 Svobodny pr., 660041, Krasnoyarsk, Russia \\ *e-mail: sonique@icm.krasn.ru
}

\begin{abstract}
This work deals with the transient separation of a multicomponent mixture in a cylindrical thermogravitational column. The model describing the evolution of separation in vertical direction is derived and solved analytically. It is assumed that the profiles of velocity and temperature in radial direction are established and the forgotten effect as well as vertical diffusion in the column are ignored. The dependence of separation and characteristic transient time on the ratio of inner and outer cylinders radii $\delta$ is obtained. The transient time tends to diffusion time along the column height when $\delta$ approaches zero, while it reduces to the transient time for a flat-plate column when $\delta$ approaches unity. The analytical results are compared with two-dimensional numerical simulation for binary and ternary mixtures, and a good agreement is observed. It is concluded that for typical liquid mixtures, the decrease of radii ratio between inner and outer cylinders results in a slight increase of separation and in a significant increase of transient time.
\end{abstract}

Keywords: multicomponent mixture, heat and mass transfer, diffusion, thermal diffusion, transport coefficients, thermogravitational column.

\section{Introduction}

The transport of species in a mixture, which leads to the formation of concentration gradients in response to the temperature gradient, is known as thermal diffusion, or the Soret effect [1]. This effect plays an important role in a number of natural and technological processes, such as distribution of components in hydrocarbon reservoirs [2] and nuclear reactors [3], species transport through the cell membranes [4], isotope separation in liquid and gaseous mixtures [5], characterization and separation of polymers [6], etc.

To describe the mass transfer induced by thermal diffusion in a mixture, one needs to know the transport coefficients, which relate the diffusive fluxes of components to the thermal and compositional gradients. Measurements of the Soret, diffusion and thermodiffusion coefficients are performed for binary [7-11] and ternary [12,13] mixtures using different experimental techniques in ground and weightlessness conditions $[14,15]$. Measurements for ternary and higher systems are much more complicated than for binary ones. Thus, some experimental data were obtained for binary pairs of three liquids $[16,17]$ in order to investigate the thermal diffusion in ternary mixture on the basis of the binary dependencies. Different theoretical approaches for calculating the thermal diffusion coefficients are developed nowadays and extended to the case of ternary and higher systems. Evaluation of the results against the experimental data helps to improve the developed approaches for calculating thermal diffusion coefficients [18], although the mechanisms of thermal diffusion phenomena in liquids is not fully understood yet. The extensive reviews of modern techniques and the results of experimental and theoretical investigation of the thermal diffusion (Soret) effect are presented in [19-21]. 
A well established technique for measurement of thermal diffusion coefficients is a thermogravitational column (TGC) [22]. In this apparatus, the mixture is placed into a closed container between two vertical walls (plane or concentric cylinders) with different temperatures. The horizontal Soret separation combined with the vertical convective current due to buoyancy leads to an enhanced component separation between the ends of the column. The thermal diffusion coefficients are determined from the vertical concentration gradients in the steady state. Reliable measurements can only be made when the vertical convective flow in the column is stable. The gravitational instabilities can be induced by negative thermal diffusion (when the heavier component is accumulated in the upper part of the column). However, it was experimentally shown by Bou-Ali et al $[23,24]$ that for binary mixtures with negative thermal diffusion coefficients in a cylindrical column, the potentially unstable convective flow can be stable in some range of parameters. This result still lacks clear theoretical explanation.

The TGC theory of binary mixtures in flat-plate columns was generalised to multicomponent mixtures by Haugen and Firoozabadi [25]. Ryzhkov and Shevtsova [26] also extended the TGC theory to multicomponent case using the so-called separation ratios. The stability of stationary flow of a multicomponent mixture in the flat-plate column was investigated in the subsequent works $[27,28]$. The influence of temperature and concentration dependence of diffusion and thermal diffusion coefficients on the stationary separation was studied by Labrosse [29]. One of the first investigations of the curvature effect on mass transfer in a binary mixture in a cylindrical column was performed by Yeh [30]. The stationary separation of a binary mixture in a layer between concentric cylinders was studied by Ryzhkov in [31] and the multicomponent mixture separation was subsequently analyzed by Kozlova and Ryzhkov in [32]. In the latter work, it was found that the vertical separation in the column increases with decreasing the ratio of cylinders radii.

The procedure for extracting the diffusion and thermal diffusion coefficients from transient measurements in the column was suggested in [33,34]. One of the first numerical studies of transient behavior of thermogravitational columns was presented in [35]. The author claimed that the numerical analysis of thermal diffusion columns should be useful for planning experiments and interpreting experimental data. The influence of density composition dependence on the transient separation of a binary liquid mixture ("the forgotten effect") was studied in [36]. In that work, it was found that the forgotten effect can greatly affect the separation process.

In this paper, we develop the theory of transient separation in a cylindrical column filled with a multicomponent mixture. The analytical solution describing the transient separation is derived in cylindrical coordinates. The effect of curvature and the ratio of concentric cylinders radii on the separation process and its duration is analyzed. The analytical and numerical solutions for transient separation are compared for binary and ternary mixtures.

The paper is organized as follows. In Section 2, the problem statement including governing equations and imposed conditions is presented. The analytical solution describing transient separation in a cylindrical TGC is derived in Section 3. The separation evolution and its characteristics are discussed in Section 4.

\section{The problem statement}

Let us investigate the transient separation of a multicomponent mixture in a cylindrical thermogravitational column. The considered mixture has $n$ components, and component $n$ is chosen as a solvent. The mixture composition is described by the vector of mass fractions $\boldsymbol{C}=\left(C_{1}, \ldots, C_{n-1}\right)^{T}$ given that $C_{1}+\ldots+C_{n}=1$.

In a closed column, the components are driven by thermal and compositional gradients. 
The diffusive flux $\boldsymbol{J}_{i}$ of component $i$ in the $\mathrm{n}-$ component mixture is written as follows

$$
\boldsymbol{J}_{i}=-\rho_{0}\left(\sum_{j=1}^{n-1} D_{i j} \nabla C_{j}+D_{T i} \nabla T\right)
$$

Here $T$ is temperature, $\rho_{0}$ is mixture density, $D_{i j}$ are the diffusion coefficients, and $D_{T i}$ are the thermal diffusion coefficients of components. Only $n-1$ fluxes are independent since $\sum_{i=1}^{n} \boldsymbol{J}_{i}=0$.

We suppose that the deviations of temperature $T$ and composition $\boldsymbol{C}$ from the mean constant values $T_{0}$ and $\boldsymbol{C}_{0}$ are small. Accordingly, we apply the Oberbeck-Boussinesq approximation with the equation of state $[26,32]$

$$
\rho=\rho_{0}\left(1-\beta_{T}\left(T-T_{0}\right)-\sum_{i=1}^{n-1} \beta_{i}\left(C_{i}-C_{i 0}\right)\right)=\rho_{0}\left(1-\beta_{T}\left(T-T_{0}\right)-\boldsymbol{I} \cdot B\left(\boldsymbol{C}-\boldsymbol{C}_{0}\right)\right)
$$

where $\boldsymbol{I}=(1, \ldots, 1)$ and $B=\operatorname{diag}\left(\beta_{1}, \ldots, \beta_{n-1}\right)$ is the diagonal matrix of solutal expansion coefficients. For the considered model, the density varies in the body force term only. Thus, the governing equations of motion and heat/mass transfer are as follows:

$$
\begin{aligned}
& \frac{\partial \boldsymbol{u}}{\partial t}+(\boldsymbol{u} \cdot \nabla) \boldsymbol{u}=-\rho_{0}^{-1} \nabla p+\nu \nabla^{2} \boldsymbol{u}-\mathbf{g}\left(\beta_{T}\left(T-T_{0}\right)+\boldsymbol{I} \cdot B\left(\boldsymbol{C}-\boldsymbol{C}_{0}\right)\right), \\
& \frac{\partial T}{\partial t}+(\boldsymbol{u} \cdot \nabla) T=\chi \nabla^{2} T \\
& \frac{\partial \boldsymbol{C}}{\partial t}+(\boldsymbol{u} \cdot \nabla) \boldsymbol{C}=D \nabla^{2} \boldsymbol{C}+\boldsymbol{D}_{\boldsymbol{T}} \nabla^{2} T \\
& \nabla \cdot \boldsymbol{u}=0 .
\end{aligned}
$$

Here $\boldsymbol{x}=(x, y, z)$ is the vector of coordinates, $\boldsymbol{u}=(u, v, w)$ is the velocity vector, $\mathbf{g}=$ $(0,0,-\mathrm{g})$ is the vector of gravitational acceleration, $p=P-\rho_{0} \mathbf{g} \cdot \boldsymbol{x}$ is the difference between the total and hydrostatic pressure, $D=\left\{D_{i j}\right\}$ is the matrix of $(n-1)^{2}$ diffusion coefficients, and $\boldsymbol{D}_{\boldsymbol{T}}=\left(D_{T 1}, \ldots, D_{T, n-1}\right)$ is the vector of thermal diffusion coefficients. The values of kinematic viscosity $\nu$ and thermal diffusivity $\chi$ are constant and correspond to the mean temperature and composition.

Let us use the cylindrical coordinates $(r, \varphi, z)$. The considered cylindrical TGC is the layer of width $L$ between two co-axial cylinders of height $2 h$ with radii $r_{1}$ and $r_{2}$ of inner and outer cylinders, so $L=r_{2}-r_{1}$ (see Fig. 1). The walls are maintained at different constant temperatures $T_{0} \pm \Delta T / 2$ in such a way that the inner cylinder is heated.

At the moment $t=0$, the initial component concentration is $\boldsymbol{C}=\boldsymbol{C}_{0}$. For $t \geq 0$, at the cylindrical walls we impose no-slip conditions and the absence of diffusive fluxes, and specify the temperature values:

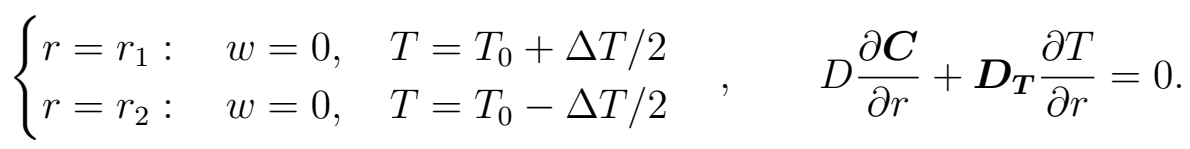

The top and bottom walls are thermally insulated and no-slip conditions and zero diffusive flux are imposed on them:

$$
z= \pm h: \quad w=0, \quad \frac{\partial T}{\partial z}=0, \quad D \frac{\partial \boldsymbol{C}}{\partial z}+\boldsymbol{D}_{\boldsymbol{T}} \frac{\partial T}{\partial z}=0 .
$$




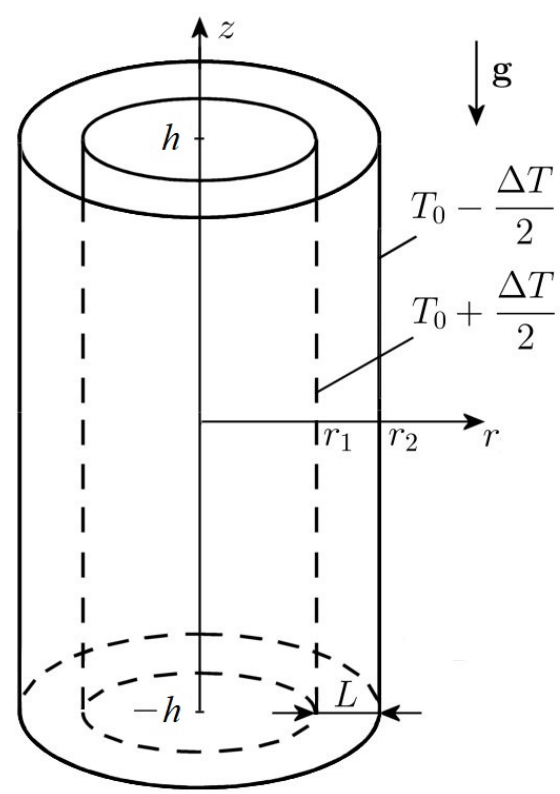

Figure 1: The geometry of a cylindrical thermogravitational column.

\section{Transient separation in the column}

\subsection{Derivation of transport equation}

In this section, we will derive the model for transient mixture separation in the vertical direction of the column. The separation will be described by the cross-sectionally averaged concentration depending on time $t$ and vertical coordinate $z$. The analytical solution of the model will be constructed in the form of infinite series.

To derive the model equation and corresponding initial and boundary conditions, a number of assumptions is introduced. The influence of composition on density, i.e. the forgotten effect, is ignored. This assumption dates back to the Furry-Jones-Onsager (FJO) theory [37], and is quite reasonable for typical liquid mixtures [38]. The forgotten effect can be important for colloidal suspensions [39,40] or near-critical mixtures [41], which lie outside the scope of this study. The convective flow in the column is assumed axisymmetric, because we consider small temperature difference between the walls, at which convection is stable, and there is no angular velocity $[22,26,27]$. This assumption is valid for both stationary and transient separation processes. On the walls, there is no vertical temperature gradient. As the ratio $2 h / L$ is large (of order $10^{2}$ and higher), the flow is strictly vertical except the top and bottom walls of the column. Taking into account zero radial velocity and incompressibility of the mixture, the continuity implies that the vertical velocity varies in the radial direction only, i.e. $\boldsymbol{u}=(0,0, w(r))$. We also assume that the stationary profiles of temperature and velocity in the column cross-section are established since viscous and thermal characteristic times are much smaller than the diffusion characteristic time for liquid mixtures. Then Eqs. (2) become

$$
\begin{aligned}
& \rho_{0}^{-1} \frac{\partial p}{\partial z}-\nu \nabla^{2} w-g\left(\beta_{T}\left(T-T_{0}\right)\right)=0, \\
& \chi \nabla^{2} T=0 \\
& \frac{\partial \boldsymbol{C}}{\partial t}+w \frac{\partial \boldsymbol{C}}{\partial z}=D \nabla^{2} \boldsymbol{C}+\boldsymbol{D}_{\boldsymbol{T}} \nabla^{2} T
\end{aligned}
$$


where the following notation is used:

$$
\nabla^{2}=\frac{1}{r} \frac{\partial}{\partial r}\left(r \frac{\partial}{\partial r}\right)+\frac{\partial^{2}}{\partial z^{2}}
$$

When the separation process reaches its steady state, the time derivative $\partial \boldsymbol{C} / \partial t$ in Eq. (7) vanishes. The solution of the stationary problem was found in [32]. In this work, we assume that the transient separation in the column is quasi-steady. Since the column gap $L$ is much smaller than its height $h$, the quasi-steady thermal and compositional fields in the column cross-section are established much faster than any appreciable separation in vertical direction can occur. It means that the time derivative $\partial \boldsymbol{C} / \partial t$ in Eq. (7) is negligible in comparison with other terms and it finally vanishes at the steady state [33]. This assumption may be violated for small times, but during the most part of transient process it is quite reasonable [33]. Thus, we obtain the mass balance equation for all components:

$$
w \frac{\partial \boldsymbol{C}}{\partial z}=\frac{1}{r} \frac{\partial}{\partial r}\left(r\left(D \frac{\partial \boldsymbol{C}}{\partial r}+\boldsymbol{D}_{\boldsymbol{T}} \frac{\partial T}{\partial r}\right)\right) .
$$

Note that in this equation the concentration $\boldsymbol{C}$ depends on time $t$. We also assume the vertical concentration gradients $\partial \boldsymbol{C} / \partial z$ are independent of $r$. Introducing the stream function by

$$
\Phi=-\int_{r_{1}}^{r} w r d r, \quad \Phi\left(r_{1}\right)=\Phi\left(r_{2}\right)=0, \quad w=-\frac{1}{r} \frac{\partial \Phi}{\partial r}
$$

and integrating Eq. (9) from $r_{1}$ to $r$ using boundary conditions (4), (10), we obtain

$$
-\Phi \frac{\partial \boldsymbol{C}}{\partial z}=\left(D \frac{\partial \boldsymbol{C}}{\partial r}+\boldsymbol{D}_{\boldsymbol{T}} \frac{\partial T}{\partial r}\right) r .
$$

For component $i$, the total vertical flux is the sum of diffusive and convective fluxes (see (1)). To obtain the net vertical flux $\boldsymbol{J}_{z}=\left(J_{1 z}, \ldots, J_{n-1, z}\right)$, we integrate the vertical component of the total flux through the column cross section of the area $S=\pi\left(r_{2}^{2}-r_{1}^{2}\right)$ :

$$
\boldsymbol{J}_{z}=\rho \int_{0}^{2 \pi} \int_{r_{1}}^{r_{2}}\left(w \boldsymbol{C}-D \frac{\partial \boldsymbol{C}}{\partial z}\right) r d r d \varphi
$$

Integration of the first term by $r$ is performed by parts with the use of Eq. (10) and Eq. (11), from which we use the expression for $\partial \boldsymbol{C} / \partial r$ :

$$
\int_{r_{1}}^{r_{2}} w \boldsymbol{C} r d r=\int_{r_{1}}^{r_{2}}\left(-\frac{1}{r} \frac{\partial \Phi}{\partial r} \boldsymbol{C}\right) r d r=-\left.\Phi \boldsymbol{C}\right|_{r_{1}} ^{r_{2}}+\int_{r_{1}}^{r_{2}} \Phi \frac{\partial \boldsymbol{C}}{\partial r} r d r=-D^{-1}\left(\int_{r_{1}}^{r_{2}} \frac{\Phi^{2}}{r} \frac{\partial \boldsymbol{C}}{\partial z}+\Phi \boldsymbol{D}_{\boldsymbol{T}} \frac{\partial T}{\partial r}\right) d r .
$$

The steady-state radial temperature gradient $\partial T / \partial r$ for a cylindrical column is derived from the temperature distribution obtained in [32]:

$$
T(r)=\Delta T \frac{\ln \left(r\left(1-r_{1} / r_{2}\right) / L \sqrt{r_{1} / r_{2}}\right)}{\ln \left(r_{1} / r_{2}\right)}, \quad \frac{\partial T}{\partial r}=\frac{\Delta T}{r \ln \left(r_{1} / r_{2}\right)} .
$$

Along with the above assumption that concentration gradients $\partial \boldsymbol{C} / \partial z$ does not depend on $r$, we finally derive the net vertical flux in the form

$$
\boldsymbol{J}_{z}=2 \pi \rho\left(D^{-1} \boldsymbol{D}_{\boldsymbol{T}} H-\left(K D^{-1}+D \frac{\left(r_{2}^{2}-r_{1}^{2}\right)}{2}\right) \frac{\partial \boldsymbol{C}}{\partial z}\right) .
$$


In Eq. (13), the following notation is used:

$$
H=-\frac{\Delta T}{\ln \left(r_{1} / r_{2}\right)} \int_{r_{1}}^{r_{2}} \frac{\Phi}{r} d r, \quad K=\int_{r_{1}}^{r_{2}} \frac{\Phi^{2}}{r} d r .
$$

Introducing the concentration averaged over the column cross section

$$
\overline{\boldsymbol{C}}(t, z)=\frac{1}{\pi\left(r_{2}^{2}-r_{1}^{2}\right)} \int_{0}^{2 \pi} \int_{r_{1}}^{r_{2}} \boldsymbol{C} r d r d \varphi
$$

we can now obtain the mass balance equation in the vertical direction in terms of the average concentration. This equation is given by

$$
\rho \frac{\partial \bar{C}_{i}}{\partial t}=-\frac{\partial \boldsymbol{J}_{z}}{\partial z}
$$

Substituting the net vertical flux (13) into Eq. (15), we obtain

$$
\frac{\partial \overline{\boldsymbol{C}}}{\partial t}=\left(\frac{2 K}{\left(r_{2}^{2}-r_{1}^{2}\right)} D^{-1}+D\right) \frac{\partial^{2} \overline{\boldsymbol{C}}}{\partial z^{2}} .
$$

The boundary conditions on the top and bottom walls at $t \geq 0$ are zero net flux $\left(\boldsymbol{J}_{z}=0\right)$, which means that the concentration gradient at these boundaries reaches its steady-state value:

$$
z= \pm h: \quad \frac{\partial \overline{\boldsymbol{C}}}{\partial z}=\frac{\Delta \overline{\boldsymbol{C}}_{\infty}}{2 h} .
$$

Here $\Delta \bar{C}_{\infty}$ is the steady-state value of the concentration difference, which is calculated from Eq. (13) when $\boldsymbol{J}_{z}=0$ :

$$
\Delta \overline{\boldsymbol{C}}_{\infty}=2 h H\left(K E+D^{2}\left(\frac{r_{2}^{2}-r_{1}^{2}}{2}\right)\right)^{-1} \boldsymbol{D}_{\boldsymbol{T}} .
$$

Here $E$ is the unit matrix.

The initial condition transforms into

$$
t=0: \quad \bar{C}=C_{0} .
$$

\subsection{Analytical solution}

In this section, we derive the analytical solution for problem (16), (17), (19). Let us introduce the ratio of cylinders radii:

$$
\delta=\frac{r_{1}}{r_{2}}, \quad 0<\delta<1,
$$

by use of which the lateral boundaries are transformed into

$$
r_{1}=\frac{L \delta}{1-\delta}, \quad r_{2}=\frac{L}{1-\delta} .
$$

In what follows, we will need the diagonalized matrix of diffusion coefficients, which can be obtained with the help of transformation

$$
\Lambda=V^{-1} D V
$$


where $\Lambda=\operatorname{diag}\left\{\lambda_{1}, \ldots, \lambda_{n-1}\right\}$ is the diagonal eigenvalue matrix of diffusion matrix $D, V$ is the matrix, which columns are the eigenvectors $\boldsymbol{v}_{i}=\left\{v_{i 1}, \ldots, v_{1, n-1}\right\}^{T}$ of $D, i=1, \ldots, n-1$. With the use of Eq. (20) and Eq. (21), governing equation (16) transforms into

$$
\frac{\partial \overline{\boldsymbol{\omega}}}{\partial t}=\left(\frac{2 K}{L^{2}}\left(\frac{1-\delta}{1+\delta}\right) \Lambda^{-1}+\Lambda\right) \frac{\partial^{2} \overline{\boldsymbol{\omega}}}{\partial z^{2}},
$$

where the transformed concentration $\overline{\boldsymbol{\omega}}=\boldsymbol{V}^{-1} \overline{\boldsymbol{C}}$ was introduced.

The analytical solution of Eq. (22) will be found in the dimensionless form after substitution

$$
z=2 h \widetilde{z}, \quad t=t_{c i} \widetilde{t}, \quad \bar{\omega}_{i}=\Delta \bar{C}_{i \infty} \widetilde{\omega}_{i},
$$

in which the characteristic time $t_{c i}$ is given by

$$
t_{c i}=4 h^{2}\left(\frac{2 K}{L^{2} \lambda_{i}}\left(\frac{1-\delta}{1+\delta}\right)+\lambda_{i}\right)^{-1}, \quad i=1, \ldots, n-1,
$$

and $\Delta \bar{C}_{\infty}$ is taken from Eq. (18).

Along with the characteristic time, the relaxation time is introduced as

$$
t_{r i}=\frac{t_{c i}}{\pi^{2}}
$$

Finally, Eq. (22) converts into

$$
\frac{\partial \widetilde{\boldsymbol{\omega}}}{\partial t}=\frac{\partial^{2} \widetilde{\boldsymbol{\omega}}}{\partial z^{2}}
$$

The boundary condition (17) becomes

$$
\widetilde{z}= \pm \frac{1}{2}: \quad \frac{\partial \widetilde{\omega}}{\partial \widetilde{z}}=1
$$

The initial condition takes the form

$$
\widetilde{t}=0: \quad \widetilde{\boldsymbol{\omega}}_{0}=\frac{\boldsymbol{V}^{-1} \overline{\boldsymbol{C}}_{0}}{\Delta \overline{\boldsymbol{C}}_{\infty}} .
$$

The problem (24)-(26) is solved by the Fourier method of separation of variables. The boundary condition (25) is transformed beforehand to the homogeneous condition by subtraction of the linear concentration profile in the steady state from the sought-for function. In dimensionless form, we have

$$
\widetilde{z}^{\prime}=\widetilde{z}+\frac{1}{2}: \quad \widetilde{\boldsymbol{\omega}}^{\prime}(\widetilde{z}, t)=\widetilde{\boldsymbol{\omega}}(\widetilde{z}, t)-\left(\widetilde{z}+\widetilde{\boldsymbol{\omega}}_{0}\right),
$$

and finally arrive to the following conditions:

$$
\widetilde{z}^{\prime}=0,1: \quad \frac{\partial \widetilde{\boldsymbol{\omega}}^{\prime}}{\partial \widetilde{z}}=0 .
$$

As a result, we obtain the concentration in dimensional form as follows

$$
\begin{aligned}
\bar{C}_{i}(z, t)=\bar{C}_{0 i}+2 h H(K E & \left.+D^{2} L^{2}\left(\frac{1+\delta}{1-\delta}\right)\right)^{-1}\left(\frac{z}{2 h} D_{T i}+\right. \\
+ & \left.\sum_{k=0}^{\infty} \frac{4}{\pi^{2}(2 k+1)^{2}} \cos \left(\frac{(z+h)(2 k+1) \pi}{2 h}\right) \exp \left(-\frac{(2 k+1)^{2} t}{t_{r i}}\right)\right) .
\end{aligned}
$$


As the forgotten effect is ignored, coefficients $H$ and $K$ defined in Eq. (14) are calculated by means of the stream function. For a cylindrical column, the latter was found in Eq. [32]. In dimensional form, we have

$$
\Phi=\operatorname{Gr} L \nu\left(\left(\frac{\ln (r / L)}{16 \ln \delta}-\frac{5}{64 \ln \delta}+c_{1}\right)\left(\frac{r}{L}\right)^{4}+\left(c_{2} \ln \left(\frac{r}{L}\right)+c_{3}\right)\left(\frac{r}{L}\right)^{2}+c_{4}\right),
$$

where $\mathrm{Gr}=\mathrm{g} \beta_{T} \Delta T L^{3} / \nu^{2}$ is the Grashof number and constant coefficients $c_{i}$ are given by

$$
\begin{aligned}
c_{1}= & \left(\left(4\left(\delta^{4}-1\right) \ln \delta-4\left(1-\delta^{2}\right)^{2}\right) \ln (1-\delta)-4 \delta^{4} \ln ^{2} \delta+\left(7 \delta^{4}-4 \delta^{2}-3\right) \ln \delta-\right. \\
& \left.4\left(1-\delta^{2}\right)^{2}\right)(\delta-1)^{4} S^{-1}, \\
c_{2}= & 2\left(\delta^{4}-4 \delta^{2} \ln \delta-1\right)(\delta-1)^{3}(\delta+1) S^{-1}, \\
c_{3}= & \left(2\left(\left(\left(4 \delta^{2}-4 \delta^{4}\right) \ln \delta+\delta^{6}-\delta^{4}-\delta^{2}+1\right) \ln (1-\delta)+4 \delta^{4} \ln ^{2} \delta-\left(3 \delta^{4}-4 \delta^{2}+1\right) \ln \delta\right)-\right. \\
& \left.\left(\delta^{4}-4 \delta^{2} \ln \delta-1\right)\left(\delta^{2}-1\right)\right)(\delta-1)^{2} S^{-1}, \\
c_{4}= & \left(\delta^{4}-(2 \delta \ln \delta-1)^{2}\right) \delta^{2} S^{-1}, \\
S= & 64(\delta-1)^{5}(\delta+1)\left(\delta^{2} \ln \delta-\delta^{2}+\ln \delta+1\right) \ln \delta .
\end{aligned}
$$

The average concentration difference between the column ends is defined as

$$
\Delta \bar{C}_{i}(t)=\bar{C}_{i}(h, t)-\bar{C}_{i}(-h, t), \quad i=1, \ldots, n-1 .
$$

\subsection{Characteristic time}

Let us now re-write Eq. (23) as follows

$$
t_{c i}=4 h^{2}\left(\frac{2 \nu^{2} \mathrm{Gr}^{2}}{\lambda_{i}} K(\delta)+\lambda_{i}\right)^{-1}
$$

where $K(\delta)$ is the function dependent on $\delta$ only. According to Eqs. (14) and (27), it has the form

$$
K(\delta)=\frac{1-\delta}{1+\delta} \int_{(1-\delta)^{-1}}^{\delta(1-\delta)^{-1}} \frac{\widetilde{\Phi}^{2}}{\widetilde{r}} d \widetilde{r},
$$

for which $\widetilde{\Phi}(\widetilde{r}, \delta)=\Phi(r / L, \delta) /(\operatorname{Gr} L \nu)$ from $(27)$.

As $K(\delta) \rightarrow 0$ when $\delta \rightarrow 0$, the limit of $t_{c i}$ is $4 h^{2} / \lambda_{i}$, which is the diffusion time at the length $2 h$ corresponding to the eigenvalue $\lambda_{i}$. When $\delta \rightarrow 1$, Eq. (29) reduces to the case of a flat-plate column

$$
t_{c i}=4 h^{2}\left(\frac{\nu^{2} \mathrm{Gr}^{2}}{9 ! \lambda_{i}}+\lambda_{i}\right)^{-1} .
$$

For typical mixtures in TGC, $\nu \sim 10^{-6}, \lambda_{i} \sim 10^{-9}, \mathrm{Gr} \sim 10$, so the ratio of the first term to the second term in parentheses is $\nu^{2} \mathrm{Gr}^{2} /\left(9 ! \lambda_{i}^{2}\right) \sim 10^{2}$. In this case, the second term $\lambda_{i}$ can be neglected and Eq. (30) becomes

$$
t_{c i}=9 ! \lambda_{i}\left(\frac{2 h \nu}{g \Delta T \beta_{T} L^{3}}\right)^{2}, \quad i=1, \ldots, n-1 .
$$

For binary mixture with $n=2$ and diffusion coefficient $D \equiv \lambda_{i}$, formula (31) recovers the characteristic time reported earlier in [10]. 
Table 1: The physical properties of ethanol - water mixture at the temperature of $T_{0}=25^{\circ} \mathrm{C}[42-45]$. Water is the solvent. The mass fraction of ethanol is $C_{0}=0.5$.

\begin{tabular}{llll}
\hline \hline Property & Value & Property & Value \\
\hline$\rho_{0}, \mathrm{~kg} / \mathrm{m}^{3}$ & 909.9 & $\beta$ & 0.246 \\
$\kappa, \mathrm{W} /(\mathrm{m} \cdot \mathrm{K})$ & 0.313 & $D, 10^{-10} \mathrm{~m}^{2} / \mathrm{s}$ & 3.620 \\
$c_{p}, \mathrm{~J} / \mathrm{K}$ & 3849 & $D_{T}^{\prime}, 10^{-12} \mathrm{~m}^{2} /(\mathrm{s} \cdot \mathrm{K})$ & -1.870 \\
$\beta_{T}, 10^{-3} \mathrm{~K}^{-1}$ & 0.882 & $\nu, 10^{-6} \mathrm{~m}^{2} / \mathrm{s}$ & 2.603 \\
\hline \hline
\end{tabular}

Table 2: The physical properties of the mixture dodecane - isobutylbenzene - tetralin at the temperature of $T_{0}=25^{\circ} \mathrm{C}[12,14,46-51]$. Component 1 is dodecane, component 2 is isobutylbenzene. The mass fraction of each component is $C_{i 0}=1 / 3, i=1,2,3$.

\begin{tabular}{llll}
\hline \hline Property & Value & Property & Value \\
\hline$\rho_{0}, \mathrm{~kg} / \mathrm{m}^{3}$ & 843.5 & $D_{11}, 10^{-10} \mathrm{~m}^{2} / \mathrm{s}$ & 6.70 \\
$\kappa, \mathrm{W} /(\mathrm{m} \cdot \mathrm{K})$ & 0.141 & $D_{12}, 10^{-10} \mathrm{~m}^{2} / \mathrm{s}$ & 0.43 \\
$c_{p}, \mathrm{~J} / \mathrm{K}$ & 1819 & $D_{21}, 10^{-10} \mathrm{~m}^{2} / \mathrm{s}$ & -1.08 \\
$\beta_{T}, 10^{-3} \mathrm{~K}^{-1}$, & 0.914 & $D_{22}, 10^{-10} \mathrm{~m}^{2} / \mathrm{s}$ & 11.10 \\
$\beta_{1}$ & 0.258 & $D_{T 1}, 10^{-12} \mathrm{~m}^{2} /(\mathrm{s} \cdot \mathrm{K})$ & -0.81 \\
$\beta_{2}$ & 0.121 & $D_{T 2}, 10^{-12} \mathrm{~m}^{2} /(\mathrm{s} \cdot \mathrm{K})$ & -0.93 \\
$\nu, 10^{-6} \mathrm{~m}^{2} / \mathrm{s}$ & 1.53 & & \\
\hline \hline
\end{tabular}

\section{Working mixtures and their physical properties}

\subsection{Binary mixture}

To investigate the transient separation in the column on the basis of obtained solution, we consider an example of ethanol-water binary mixture. The water is chosen as a solvent. The thermophysical properties of this mixture are presented in Table 1. Their values correspond to mass fraction of ethanol $C_{0}=0.5$ and the temperature of $T_{0}=25^{\circ} \mathrm{C}$. Note that for a binary mixture, the thermal diffusion coefficient is defined as $D_{T}=D_{T}^{\prime} C_{0}\left(1-C_{0}\right)$.

\subsection{Ternary mixture}

In addition to the binary system, we also consider the ternary mixture of dodecane $\left(\mathrm{C}_{12} \mathrm{H}_{26}\right)$ - isobutylbenzene $\left(\mathrm{C}_{10} \mathrm{H}_{14}\right)$ - 1,2,3,4-thetrahydronaphthalene (tetralin) $\left(\mathrm{C}_{10} \mathrm{H}_{12}\right)$, for which tetralin is chosen as the solvent. Such mixtures are common for nature deposits of hydrocarbons. For this mixture, the diffusion and thermal diffusion coefficients were measured by a number of research laboratories $[12,14]$. Its thermophysical properties are presented in Table $2[47,48]$. The mixture heat capacity and thermal conductivity were calculated as mass-weighted average of heat capacities and thermal conductivities of pure components [49-51]. The mean values of the mixture properties correspond to the average volume fractions $C_{i 0}=1 / 3, i=1,2,3$, and the temperature of $T_{0}=25{ }^{\circ} \mathrm{C}$. 


\section{Numerical simulation}

The two-dimensional numerical simulation of mixture separation is performed in ANSYS Fluent 14.5 (Academic version). We consider a rectangular domain $1 \times 500 \mathrm{~mm}$ of a longitudinal column cross-section, which corresponds to the gap width between co-axial cylinders of $1 \mathrm{~mm}$ and the height of $500 \mathrm{~mm}$. Such parameters are typical for thermogravitational columns. The studied columns have the ratios of cylinders radii $\delta=r_{1} / r_{2}=0.1,0.5$ and 0.9 . For the considered domains, several computational meshes were considered: 1) 20 and 500,2) 20 and 1000,3) 20 and 2000,3) 30 and 1000 cells in $r$ and $z$ directions, respectively. Mesh tests were performed for the ternary hydrocarbon mixture. Tests showed that steady-state concentration gradients of dodecane are $\Delta \bar{C}_{1} / \Delta z$ are equal to $0.078526,0.077902,0.077451$ and 0.077333 for meshes 1-4, respectively. Concentration gradient for meshes 3 and 4 are the closest to the theoretical gradients value 0.07725 , but the performance time doubles with the increase of cell number from 20 to 30 . Mesh 1 has the worst concentration gradient value, thus, we have chosen the mesh with $20 \times 1000$ cells.

In ANSYS Fluent, the mean temperature of both mixtures are $25^{\circ} \mathrm{C}$ and the temperature difference between the walls is $\Delta T=4^{\circ} \mathrm{C}$. The no-slip condition and zero diffusive flux of the mixture components are imposed at the side walls, zero heat flux is specified on the top and bottom walls. The problem is solved iteratively by the SIMPLEC algorithm (Semi-Implicit Method for Pressure-Linked Equations Consistent). The solver type is pressure-based and the second-order approximation scheme is used. The time step was determined according to the viscous time, which is determined as $t_{v}=L^{2} / \nu$ and equals $0.384 \mathrm{~s}$ for the binary and $0.65 \mathrm{~s}$ for the ternary mixtures, respectively. In both cases, the time step is chosen as $0.01 \mathrm{~s}$ in the beginning (until temperature and velocity profiles between the walls are developed) and $1 \mathrm{~s}$ afterwards.

For multicomponent mixtures, the equations contain a matrix of $(n-1)^{2}$ diffusion coefficients $D_{i j}$. Thus, for a ternary mixture we have a $2 \times 2$ matrix. In ANSYS Fluent, the cross diffusion coefficients of the matrix $D$ can only be set equal, which is not the case in our study. To resolve this problem, we transform the mass transfer equations to those with a diagonal diffusion matrix by transformation (21). As it was made earlier for Eq. (22), the components concentrations $\boldsymbol{C}$ and thermal diffusion coefficients $\boldsymbol{D}_{\boldsymbol{T}}$ have to be transformed also [28, 52], though the form of Navier-Stokes equations remains the same.

To make the transformation, let us introduce a complementary matrix

$$
Q=\operatorname{diag}\left\{q_{1}, \ldots, q_{n-1}\right\}, \quad q_{i}=\beta_{i}^{-1} \sum_{j=1}^{n-1} \beta_{j} v_{i j},
$$

where $\boldsymbol{v}_{i}=\left\{v_{i 1}, \ldots, v_{1, n-1}\right\}^{T}, i=1, \ldots, n-1$, are the eigenvectors of the diffusion matrix $D$ (see $(21)$ ). Then we introduce the following change of concentration and thermal diffusion coefficients

$$
\boldsymbol{C}=V Q^{-1} \widetilde{\boldsymbol{C}}, \quad \boldsymbol{D}_{\boldsymbol{T}}=V Q^{-1} \widetilde{\boldsymbol{D}}_{T}
$$

to equations (2), in which, finally, $\boldsymbol{C}, \boldsymbol{C}_{0}$, and $\boldsymbol{D}_{\boldsymbol{T}}$ are replaced by $\widetilde{\boldsymbol{C}}, \widetilde{\boldsymbol{C}}_{0}$, and $\widetilde{\boldsymbol{D}}_{T}$, respectively. We suppose that $q_{i} \neq 0$, so $\operatorname{det} Q \neq 0$ and transformation (32) is reversible.

The numerical calculations in Ansys Fluent are performed in terms of transformed variables and diagonal diffusion matrix. After that, the original concentrations $\mathrm{C}$ are restored from Eq. (32). This approach allows taking into account different cross-diffusion coefficients in the simulation. 

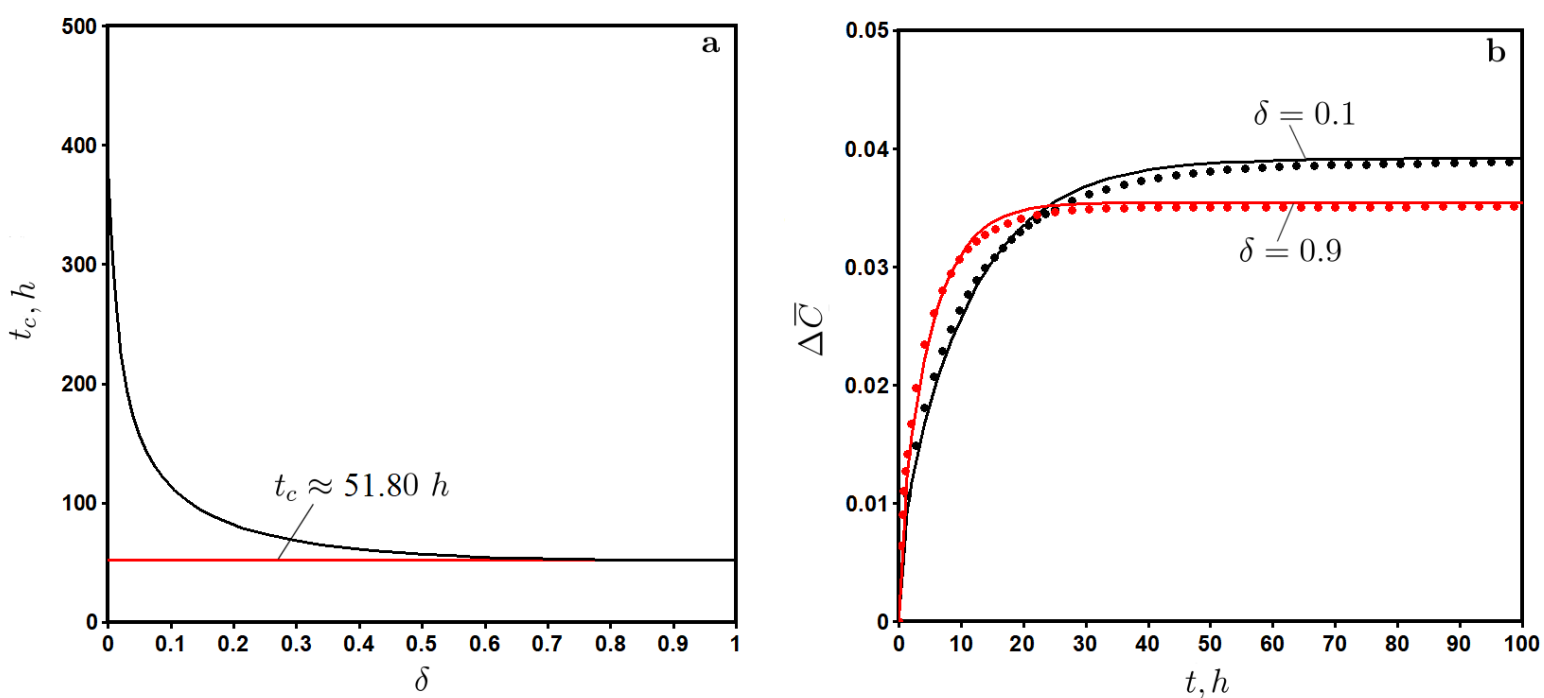

Figure 2: The dependance of characteristic time of ethanol - water mixture on the ratio of the cylinders radii $\delta$. Red line corresponds to the case of a flat-plate column. The evolution of the average concentration difference of ethanol between the column ends for the ratios of cylinder radii $\delta=0.1$ (a) and $\delta=0.1$ (b). Analytical solution (solid lines), numerical simulation (dots).

\section{Results and discussion}

\subsection{Binary mixture}

In the following, we analyse the evolution of mixture separation process. First, we evaluate the characteristic time $t_{c}$ from Eq. (29) for the binary mixture ethanol - water according to the ratio of cylinders radii $\delta$. The dependance $t_{c}(\delta)$ is shown in Fig. 2a. One can see from the picture that the time grows fast when the ratio of cylinders radii decreases. When $\delta \rightarrow 0, t_{c}$ tends to around 7962 days. In the limit of a plane column $\delta \rightarrow 1, t_{c}$ converges to the value of $51.8 \mathrm{~h}$.

As the thermal diffusion coefficient of ethanol is negative (normal thermal diffusion), ethanol goes to the hot wall. Due to the convective current, it starts accumulating in the upper part of the column. The evolution of ethanol concentration difference is shown in Fig. $2 \mathrm{~b}$ for the column with $\delta=0.1$ and $\delta=0.9$. Characteristic times for these columns are 113.7 $\mathrm{h}$ and $51.9 \mathrm{~h}$, respectively, so the results for the column with the ratio $\delta=0.9$ are close to the case of a plane column.

Let us now discuss the separation of mixtures with different thermal diffusion properties in a cylindrical column. We use the special parameter known as the separation ratio, which characterizes the ratio of density gradients induced by concentration and temperature gradients in a closed system under mechanical equilibrium. For a binary mixture, the separation ratio is defined as $\Psi=-\beta_{T}^{-1} \beta D^{-1} D_{T}$. The value of $\Psi$ is large when the contribution of concentration change due to the Soret effect is large relative to the contribution of temperature. It was shown that the mixture separation increases with the growth of $\Psi$ in a plane column [26]. For a cylindrical column, it was found that the separation becomes greater when the ratio of cylinders radii decreases and $\Psi$ is fixed [32]. Moreover, the greater the separation ratio, the larger the separation increase with the decrease of the ratio of cylinders radii. Along with this, the characteristic transient time grows as well. For the considered binary system $\Psi=0.377$ [28], which is a small value. In such a case, as it is shown in Fig. 2b, the steady-state concentration difference grows only slightly from $3.5 \%$ for $\delta=0.9$ to $3.9 \%$ for $\delta=0.1$, while the characteristic time increases more than twice. 

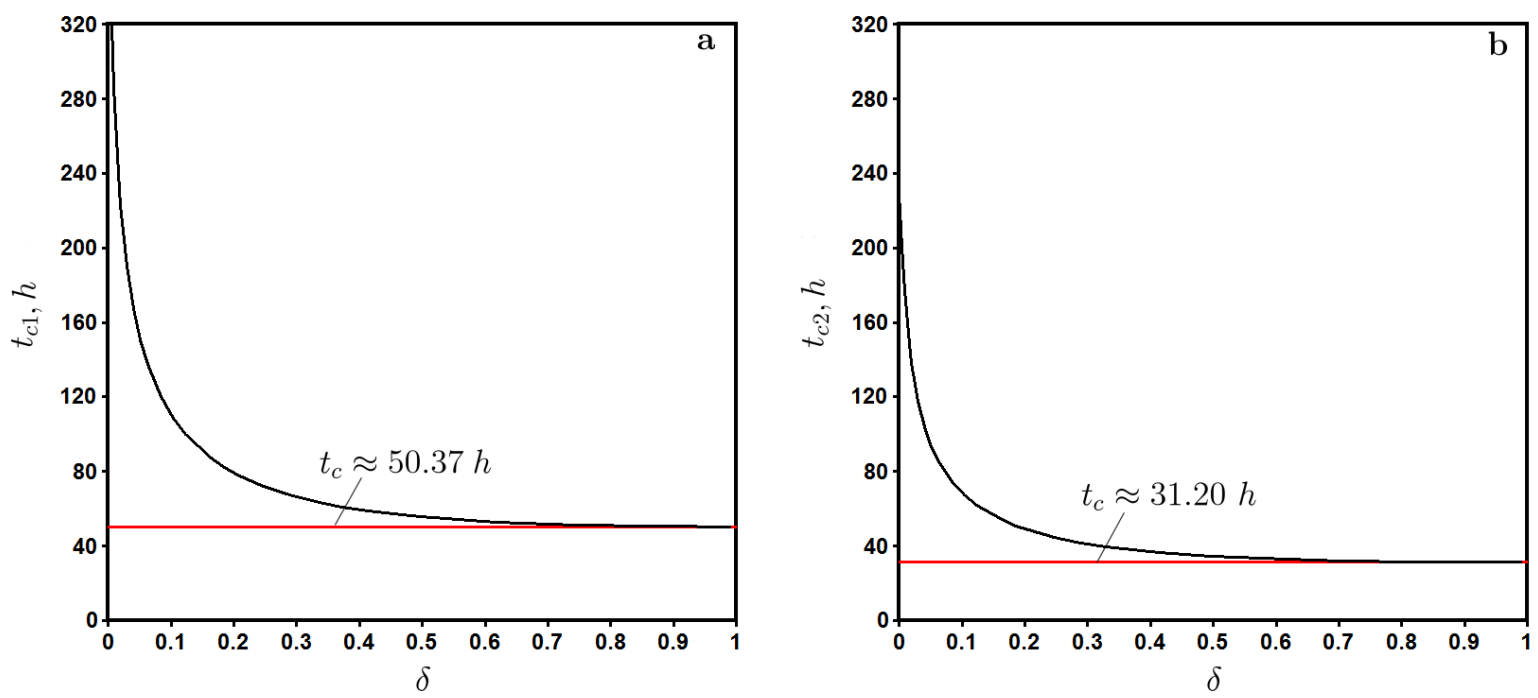

Figure 3: The dependance of characteristic times on the ratio of the cylinders radii $\delta: t_{c 1}(\mathrm{a}), t_{c 2}(\mathrm{~b})$. Red lines correspond to the case of a flat-plate column.

It is also seen from Fig. 2b that the numerical concentration difference is slightly smaller than the analytical one during time. This happens because the concentration dependance of density (the forgotten effect) is taken into account in the numerical simulation, but it is ignored in the analytical solution $[33,34]$. By the same reason, the analytical steady-state concentration difference is slightly larger than the numerical one. Thus, the impact of the forgotten effect is not significant in steady and transient regimes. Note that this conclusion is valid for mixtures with small separation ratios $\Psi$.

\subsection{Ternary mixture}

Let us analyse the evolution of dodecane - isobutylbenzene - tetralin mixture separation. We plot the characteristic times $t_{c i}$ in Fig. 3 for the considered ternary mixture using formula (29). As it is seen from the figure, $t_{c 1}(\delta)>t_{c 2}(\delta)$ and the times grow fast when the ratio of the cylinders radii $\delta$ decreases. When $\delta \rightarrow 0$, both times are around 4350 days, while for $\delta \rightarrow 1$, these times are $50.37 \mathrm{~h}$ and $31.20 \mathrm{~h}$, respectively. The latter case corresponds to a plane column.

The time evolution of the average longitudinal concentration profile is illustrated on the example of isobutylbenzene for the column with the ratio of the cylinders radii $\delta=0.1$. For this column, the characteristic times $(29)$ are $t_{c 1}=110.4 \mathrm{~h}, t_{c 2}=68.5 \mathrm{~h}$. As the thermal diffusion coefficient of isobutylbenzene is negative (normal thermal diffusion), isobutylbenzene goes to the hot wall and accumulates in the upper part of the column. The evolution process is shown in Fig. 4. The analytical and numerical solutions show a good agreement at the beginning of the separation process (up to $5 \mathrm{~h}$, Figs. 4a-c). The discrepancies of solutions (Fig. 4d) can be explained by the fact that the forgotten effect is ignored in the theoretical analysis [33,34], but the general model (mixture density varies with temperature and composition) is used for the numerical simulation. Anyway, the steady-state concentration profiles of isobutylbenzene for both approaches coincide, which is shown in Fig. 5. In this figure, the stationary profiles are plotted for the ratios of the cylinders radii $\delta=0.1$ and $\delta=0.9$. One can see that the steady-state separation slightly increases with the decrease of the ratio of cylinders radii [32]. As the thermal diffusion coefficient of dodecane is also negative, the same tendencies are valid for that component.

The evolution of average concentration difference between the column ends (28) also con- 

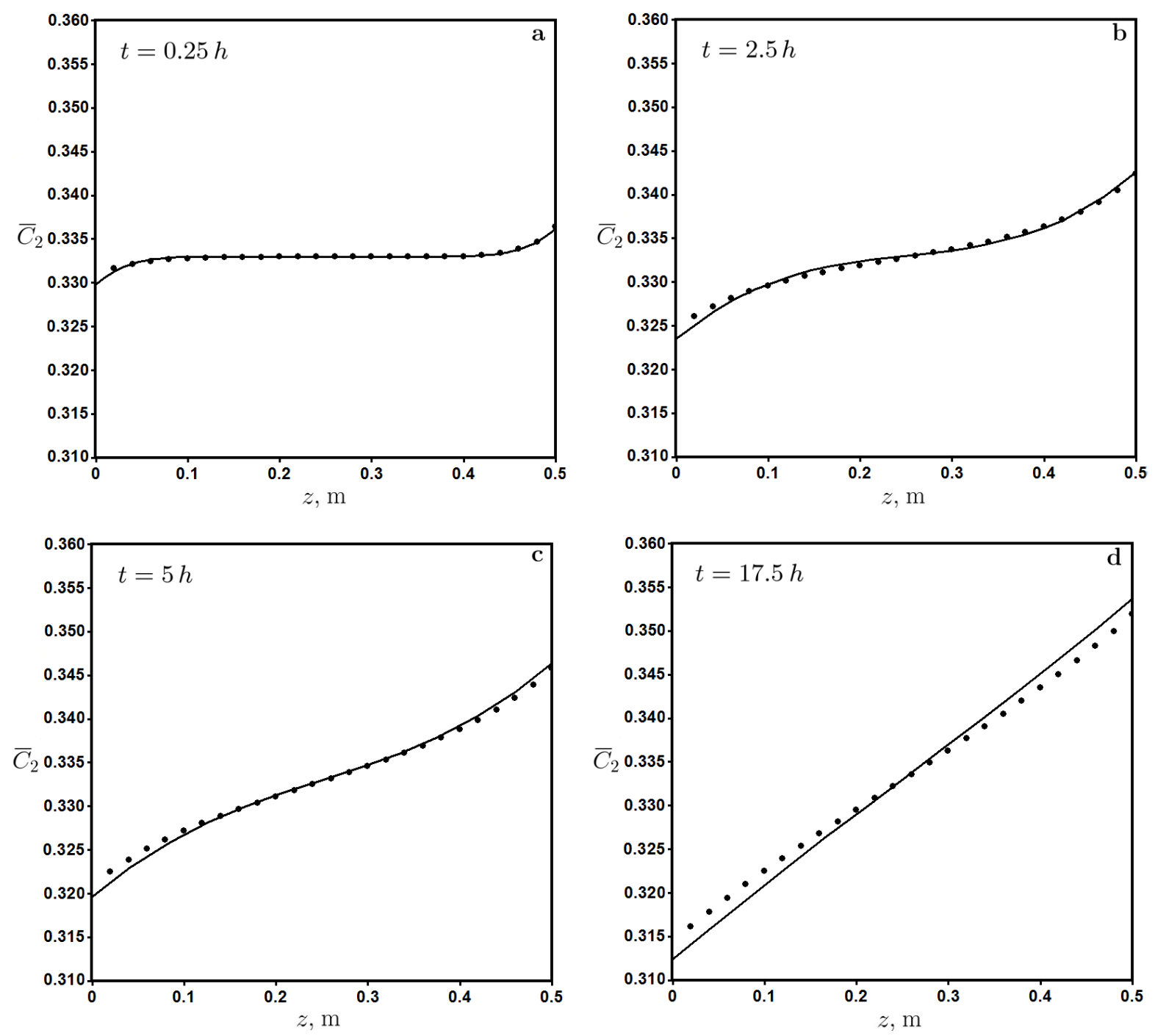

Figure 4: The evolution of the average concentration profile of isobutylbenzene along the column with the ratio of cylinders radii $\delta=0.1$. Analytical solution (solid lines), numerical simulation (dots).

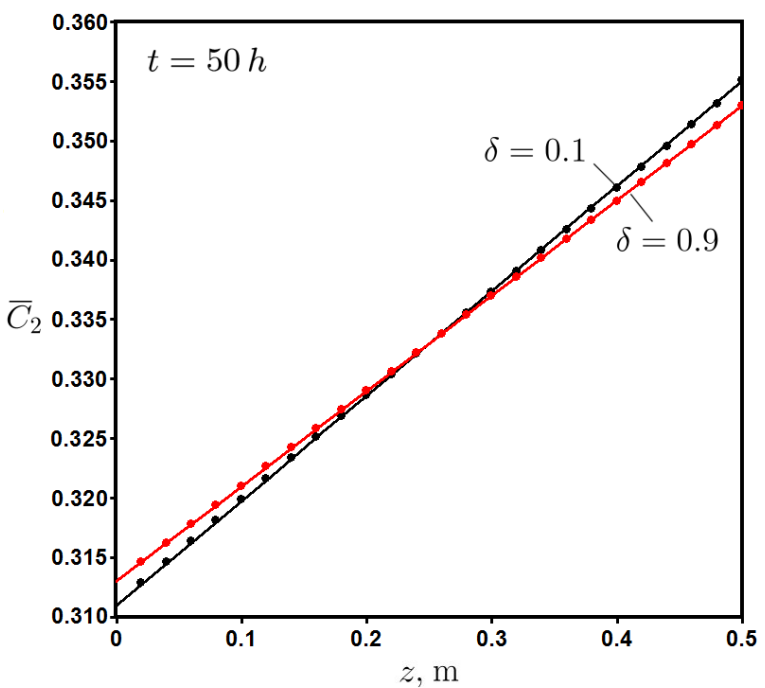

Figure 5: The steady-state average concentration profile of isobutylbenzene along the column for the ratios of the cylinders radii $\delta=0.1$ (black line) $\delta=0.9$ (red line). Analytical solution (solid lines), numerical simulation (dots). 

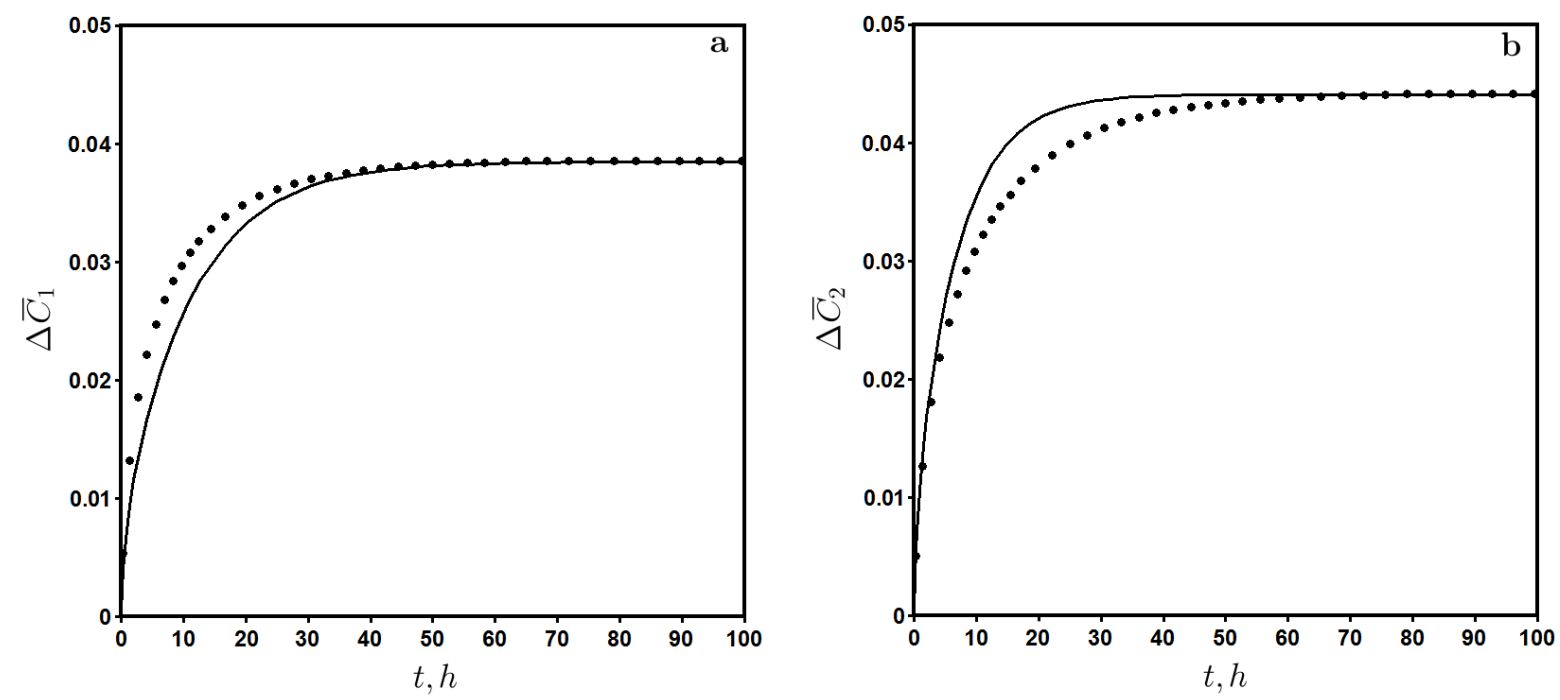

Figure 6: The evolution of the average concentration difference between the column ends for $\delta=0.1$ : dodecane (a), isobutylbenzene (b). Analytical solution (solid lines), numerical simulation (dots).
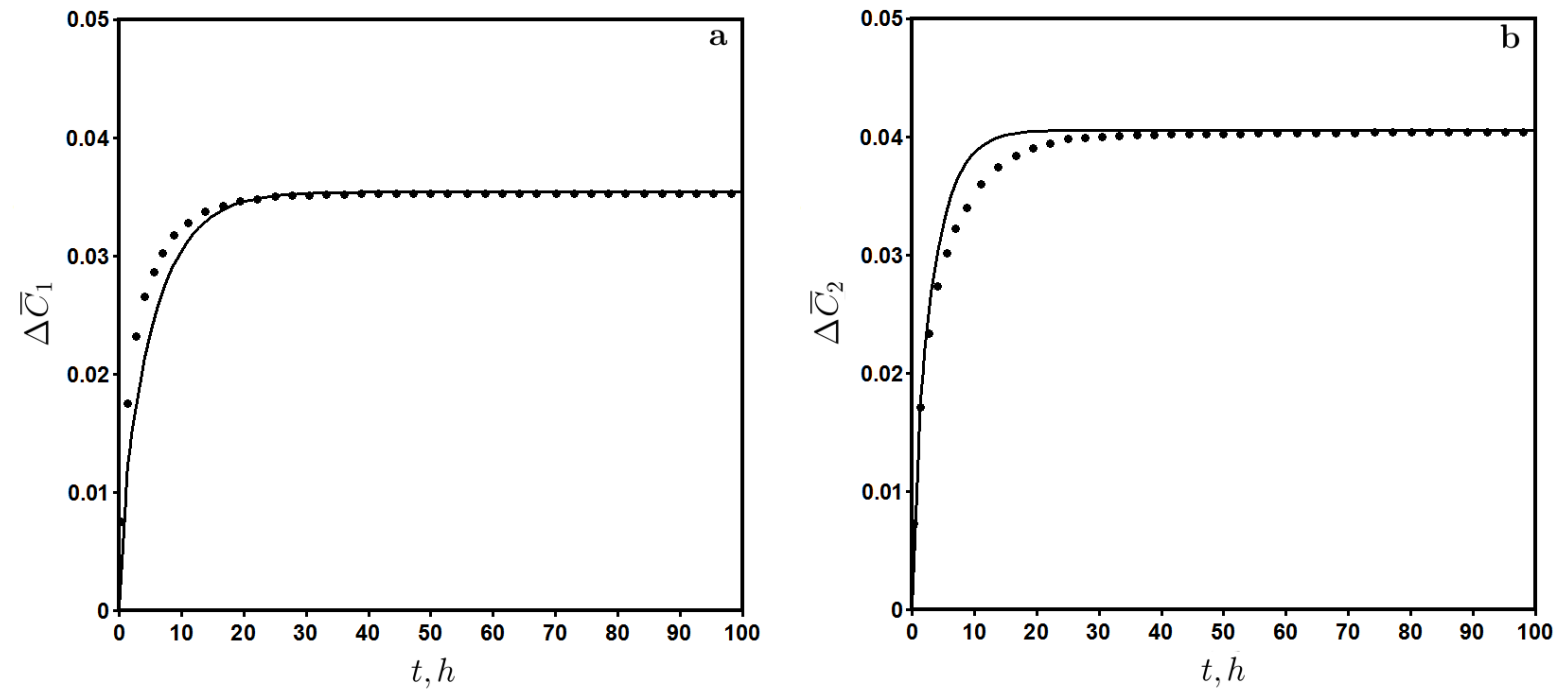

Figure 7: The evolution of the average concentration difference between the column ends for $\delta=0.5$ : dodecane (a), isobutylbenzene (b). Analytical solution (solid lines), numerical simulation (dots).
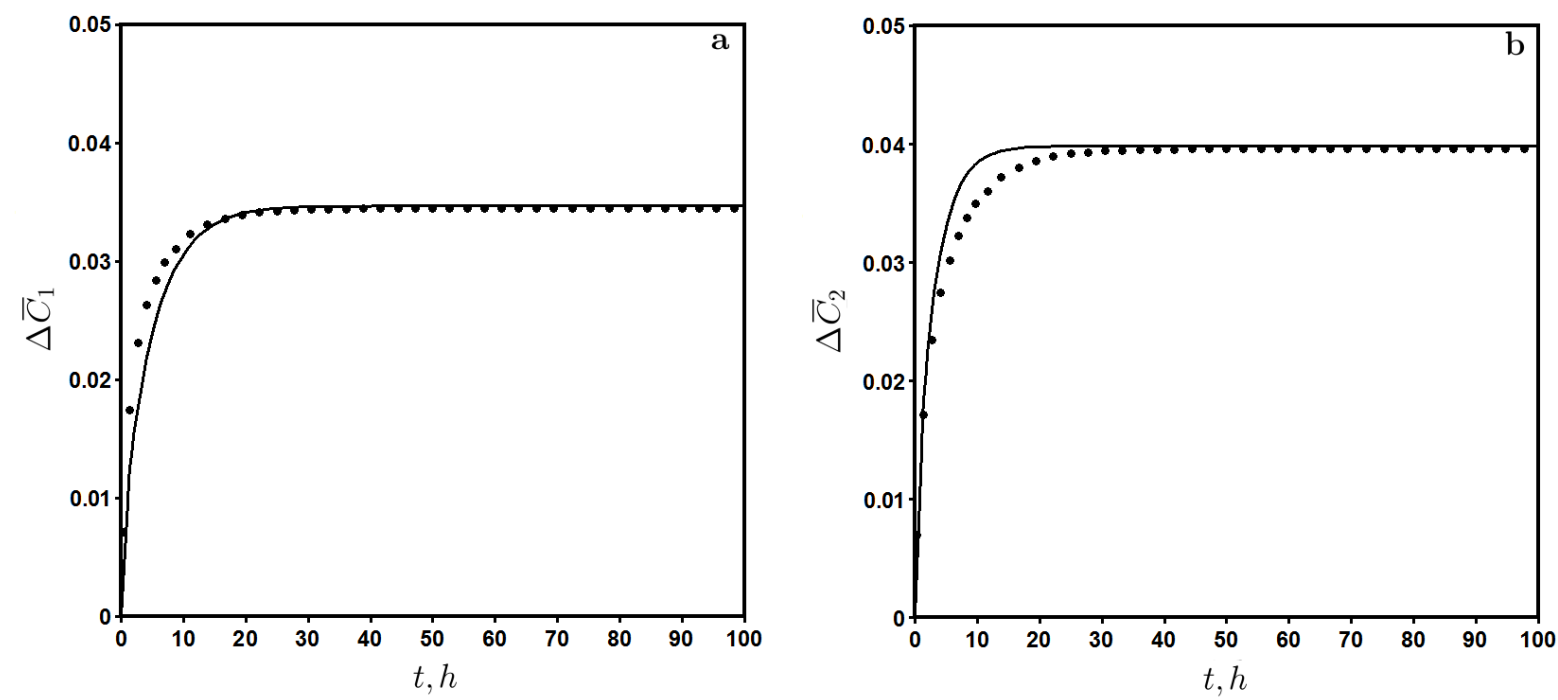

Figure 8: The evolution of the average concentration difference between the column ends for $\delta=0.9$ : dodecane (a), isobutylbenzene (b). Analytical solution (solid lines), numerical simulation (dots). 
firms the above discussion about the characteristic time and the evolution of the concentration profile. Figs. $6-8$ present the evolution of $\Delta \bar{C}_{i}$ for dodecane and isobutylbenzene in columns with the ratios of the cylinders radii $\delta=0.1, \delta=0.5$ and $\delta=0.9$, respectively. It is seen from the pictures that the steady-state value of $\Delta \bar{C}_{1}$, which corresponds to dodecane, is smaller than $\Delta \bar{C}_{2}$ for isobutylbenzene in all considered cases, because isobutylbenzene has a larger thermal diffusion coefficient. This means that the vertical separation of isobutylbenzene is slightly greater.

The steady-state values of the concentration difference for both components slightly increase with decreasing $\delta$. Let us discuss it with the help of the separation ratios, which are defined as follows $[26,32]$

$$
\psi_{i}=-\frac{\beta_{i} \nabla C_{i}}{\beta_{T} \nabla T}, \quad i=1, \ldots, n-1 .
$$

From this definition and condition of zero mass flux (1) one arrives to the relation $\boldsymbol{\psi}=$ $\left(\psi_{1}, \ldots, \psi_{n-1}\right)^{T}=-\beta_{T}^{-1} B D^{-1} \boldsymbol{D}_{T}$. The sum of separation ratios for mixture components is called the net separation ratio

$$
\Psi=\sum_{n=1}^{n-1} \psi_{i} .
$$

For the considered ternary system $\Psi=0.45$ [32], which is rather small value. As it was mentioned in the case of binary mixture, the decrease of the ratio of cylinders radii $\delta$ leads to slight separation increase, but the transient time increases significantly. For the considered ternary mixture, Figs. 5 and 7-9 show that the concentration difference is 0.034 for dodecane and around 0.04 for isobutylbenzene when $\delta=0.9$. The separation grows by $0.25-0.3 \%$ when the ratio of cylinders radii decreases from 0.9 to 0.1 , but the times $t_{c i}$ become more than twice as large.

There are also some peculiarities of the separation evolution. For all the columns, the numerical concentration profile of dodecane develops faster than the analytical solution, that is why the dotted line in Figs. 6-8a lies above the solid line. The situation is opposite for isobutylbenzene. In each case, theoretical and numerical values of $\Delta \bar{C}_{i}(t)$ eventually reach the stationary value, but, when $\delta$ decreases, numerical values become slightly smaller than the theoretical ones for both components. As it is was mentioned above, these discrepancies are explained by the forgotten effect, which is taken into account in the numerical simulation and is neglected in the analytical solution. For a mixture with small $\Psi$, this effect makes some impact during the transient state, but it is not significant in the stationary state.

From the experimental point of view, investigation of thermal diffusion in mixtures with small net separation ratios is more convenient and less time-consuming in cylindrical columns with the ratio of cylinders radii close to unity. Mixtures, which have large values of the net separation ratio, are colloidal suspensions $[39,40]$ and mixtures in the vicinity of critical conditions $[41,53]$. In such systems, the separation is rather large, and it can be further increased by decreasing $\delta$.

\section{Conclusion}

In this paper, we studied the transient multicomponent mixture separation in a cylindrical thermogravitational column. The study was performed in cylindrical coordinates in order to investigate the impact of column shape on the separation process. The parameter $\delta$ was introduced as the ratio of the inner and outer cylinders radii. With the help of this ratio, the considered problem, i.e. the parameters, equations, and obtained results, can be compared with the case of a flat-plate column. To study the separation evolution along the column, the 
concentration averaged over the column cross section was introduced. The temperature and velocity profiles in the radial direction were considered established and the diffusion in vertical direction of the column as well as the influence of composition on density in the buoyancy term were neglected. The solution describing the transient vertical concentration profile was found in the form of an infinite series. The expression for characteristic transient time was derived. The latter showed significant increase when the ratio of cylinders radii $\delta$ decreases. The limit of characteristic time when $\delta \rightarrow 0$ is the diffusion time at the total column height. For $\delta \rightarrow 1$, the time converges to that for a flat-plate column.

As an example, the binary water - ethanol mixture and the ternary mixture of dodecane - isobutylbenzene - tetralin were studied analytically and 2D numerical simulation of the transient separation was performed. In the simulation, the columns with the ratios of cylinders radii $\delta=0.1, \delta=0.5$ and $\delta=0.9$ were used. The analytical and numerical results are in a good agreement. For both mixtures, the analysis showed that the vertical separation in the column becomes slightly greater with reducing the ratio of cylinders radii. The evolution of average concentration profiles and concentration difference between the column ends revealed that the steady state separation for dodecane (isobutylbenzene) in the numerical simulation establishes faster (slower) than in the analytical solution since the latter does not take into account the forgotten effect.

\section{Acknowledgments}

The work was supported by the Russian Foundation for Basic Research Grant 15-01-03293.

\section{References}

[1] S. Wiegand. Thermal diffusion in liquid mixtures and polymer solutions. Journal of Physics: Condensed Matter, 2004. V. 16, P. 357-379.

[2] K. Ghorayeb, A. Firoozabadi. Modelling multicomponent diffusion and convection in porous media. SPEJ, 2000. V. 5, 158-171.

[3] H. Beisswenger, M. Bober, G. Schumacher. Thermodiffusion in systems $\mathrm{UO}_{2}$ and $\mathrm{CeO}_{2}$. J. Nucl. Matter, 1967. V. 21, N 1, 38-52.

[4] F.J. Bonner, L. O. Sundelöf. Thermal diffusion as a mechanism for biological transport. Z. Naturforsch. C., 1984. V. 39, N 6, 656-661.

[5] R.C. Jones, W.H. Furry. The separation of isotopes by thermal diffusion. Rev. Mod. Phys., 1946. V. 18, 151-224.

[6] M.E. Schimpf. Thermal field-flow fractionation, in Field-Flow Fractionation Handbook, edited by M. E. Schimpf, K. Caldwell, and J. C. Giddings (Wiley, New York, 2000), p. 239.

[7] P. Costesèque, J.-C. Loubet. Measuring the Soret coefficient of binary hydrocarbon mixtures in packed thermogravitational columns (contribution of Toulouse University to the benchmark test) Philosophical Magazine, 2010, V. 83, N 17-18, pp. 2017-2022.

[8] A. Khouzam, A. Mojtabi, M.-C. Charrier-Mojtabi, B. Ouattara. Species separation of a binary mixture in the presence of mixed convection. International Journal of Thermal Sciences, 2013, V. 73, pp. 18-27. 
[9] A. Mialdun, V. Yasnou, V. Shevtsova, A. Königer, W. Köhler, D. Alonso de Mezquia, and M. M. Bou-Ali. A comprehensive study of diffusion, thermodiffusion, and Soret coefficients of water-isopropanol mixtures. J. Chem. Phys., 2012, V. 136, 244512.

[10] M. M. Bou-Ali, O. Ecenarro, J. A. Madariaga, C. M. Santamaría and J. J. Valencia. Thermogravitational measurement of the Soret coefficient of liquid mixtures. J. Phys.: Condens. Matter, 1998, V. 10, pp. 3321-3331.

[11] I. Lizarraga, F. Croccolo, H. Bataller, M. M. Bou-Ali. Soret coefficient of the n-dodecanen-hexane binary mixture under high pressure. EPJ E, 2017, V. 40:36

[12] M.M. Bou-Ali, A. Ahadi, D. Alonso de Mezquia, Q. Galand, M. Gebhardt, O. Khlybov, W. Köhler, M. Larrañaga, J.C. Legros, T. Lyubimova, A. Mialdun, I. Ryzhkov, M.Z. Saghir, V. Shevtsova, and S. Van Vaerenbergh. Benchmark values for the Soret, thermodiffusion and molecular diffusion coefficients of the ternary mixture tetralin + isobutylbenzene $+\mathrm{n}-$ dodecane with 0.8-0.1-0.1 mass fraction The European physical journal E, 2015. V. 38: 30 .

[13] A. Mialdun and V. Shevtsova. Communication: New approach for analysis of thermodiffusion coefficients in ternary mixtures. J. Chem. Phys. 2013, V. 138, 161102.

[14] Khlybov O.A., Ryzhkov I.I., Lyubimova T.P. Contribution to the benchmark for ternary mixtures: measurement of diffusion and Soret coefficients in 1,2,3,4tetrahydronaphthalene, isobutylbenzene, and dodecane onboard the ISS. The European physical journal E, 2015. V. 38: 29.

[15] S. Srinivasan, M. Z. Saghir. Thermodiffusion in ternary hydrocarbon mixtures. Part 1: ndodecane/isobutylbenzene/tetralin. J. of Non-Equilibrium Thermodynamics, 2011, V. 36, N 3 .

[16] J. K. Platten, M. M. Bou-Ali, P. Costesèque, J. F. Dutrieux, W. Köhler, C. Leppla, S. Wiegand and G. Wittko. Benchmark values for the Soret, thermal diffusion and diffusion coefficients of three binary organic liquid mixtures. Phil. Mag. 2003, V. 83, N 17-18, pp. 1965-1971.

[17] M. M. Bou-Ali, J. J. Valencia, J. A. Madariaga, C. Santamaría, O. Ecenarro and J. F. Dutrieux. Determination of the thermodiffusion coefficient in three binary organic liquid mixtures by the thermogravitational method (contribution of the Universidad del País Vasco, Bilbao, to the benchmark test), Philos. Mag. 83 (2003), 2011.

[18] M. Eslamian, M. Z. Saghir. Dynamic thermodiffusion theory for ternary liquid mixtures. J. of Non-Equilibrium Thermodynamics, 2010, V. 31, N 1.

[19] P. Costesèque, A. Mojtabi, J. K. Platten. Thermodiffusion phenomena. Comptes Rendus Mècanique, 2011, V. 339, N 5, pp. 275-279.

[20] S. Srinivasan and M. Z. Saghir. Experimental approaches to study thermodiffusion - a review. Int. J. Thermal Sci. 2011, V. 50, 1125.

[21] W. Köhler and K. I. Morozov. The Soret Effect in Liquid Mixtures - A Review. J. NonEquilib. Thermodyn. 2016, V. 41(3), pp. 151197

[22] J.K. Platten. The Soret effect: A review of recent experimental results. Journal of Applied Mechanics, 2006. V. 73, 5-15. 
[23] M.M. Bou-Ali, O. Ecenarro, J.A. Madariaga, C.M. Santamaría. Stability of convection in a vertical binary fluid layer with an adverse density gradient. Phys. Rev. E. 1999. V. 59, N 1, 1250-1252.

[24] M.M. Bou-Ali, O. Ecenarro, J.A. Madariaga, C.M. Santamaría, J. Valencia. Measurement of negative Soret coefficients in a vertical fluid layer with an adverse density gradient. Phys. Rev. E. 2000. V. 62, N 1, 1420-1423.

[25] K. Haugen, A. Firoozabadi. On measurement of thermal diffusion coefficients in multicomponent mixtures. J. Chem. Phys., 2005. V. 122, 014516.

[26] I.I. Ryzhkov, V. M. Shevtsova. On thermal diffusion and convection in multicomponent mixtures with application to the thermogravitational column. Physics of Fluids, 2007. V. $19,027101$.

[27] I.I. Ryzhkov, V.M. Shevtsova V.M. Convective stability of multicomponent fluids in the thermogravitational column. Phys. Rev. E. 2009. V. 79, N 2, 026308.

[28] I.I. Ryzhkov. Thermal diffusion in mixtures: equations, symmetries, solutions and their stability. Publishing house of SB RAS, Novosibirsk, 2013 (in Russian).

[29] G. Labrosse. Free convection of binary liquid with variable Soret coefficient in thermogravitational column: The steady parallel base states. Phys. Fluids. 2003. V. 15, N 9, 2694-2727.

[30] H.M. Yeh. The effect of curvature on the transport coefficients of thermal diffusion in concentric-tube column. Separation Science, 1976. V. 11, N 5, 455-465.

[31] I.I. Ryzhkov. On double diffusive convection with Soret effect in vertical layer between co-axial cylinders, Physica D, 2006. V. 215, 191-200.

[32] S. V. Kozlova, I. I. Ryzhkov. On the separation of multicomponent mixtures in a cylindrical thermogravitational column. Physics of Fluids, 2016. V. 28, 117102.

[33] K. Haugen, A. Firoozabadi. On the unsteady-state species separation of a binary liquid mixture in a rectangular thermogravitational column. J. Chem. Phys., 2006, V. 124, 054502 .

[34] K. Haugen, A. Firoozabadi. Transient separation of multicomponent liquid mixtures in thermogravitational columns. J. Chem. Phys., 2007. V. 127, 154507.

[35] J. Powers. Transient Behavior of Thermal Diffusion Columns. Ind. Eng. Chem., 1961, V. 53 (7), pp. 577580

[36] O. Ecenarro, J.A. Madariaga, J.L. Navarro, C.M. Santamaría, J. A. Carrión, and J. M. Savirón. Non-steady-state density effects in liquid thermal diffusion columns. J. Phys.: Condens. Matter, 1989. V. 1, 9741-9750.

[37] W.H. Furry, R.C. Jones, L. Onsager. On the theory on isotope separation by thermal diffusion. Phys. Rev., 1939. V. 55, 1083-1095.

[38] J.L. Navarro, J.A. Madariaga, J.M. Savirón. The forgotten effect in liquid thermal diffusion columns. J. Phys. A: Math. Gen., 1982. V. 15, 1683-1687.

[39] R. Cerbino, S. Mazzoni, A. Vailati, and M. Giglio. Scaling behavior for the onset of convetion in colloidal suspensions. Phys. Rev. Lett., 2005, V. 94, 064501. 
[40] M.I. Shliomis, M. Souhar. Self-oscillatory convection caused by the Soret effect. Europhys. Lett., 2000. V. 49, 55.

[41] O. Ecenarro, J.A. Madariaga, J.L. Navarro, C. M. Santamaría, J.A. Carríon, and J.M. Savirón. Thermogravitational separatin and the thermal diffusion factor near critical points in binary liquid mixtures. J. Phys.: Condens. Matter, 1993. V. 5, 2289.

[42] Chemical engineer's handbook. 4th Edition / Eds. R. H. Perry, C. H. Chiltom, S. D. Kirkpatrick. N. Y.: McGraw-Hill, 1963. 1915 p.

[43] Stabnikov V. N. Distillation and rectification of ethyl alcohol. oscow: Food industry, 1969. 456 p. (in Russian).

[44] Hammond B.R., Stokes R.H. Diffusion in binary liquid mixtures. Part I. Diffusion coefficients in the system ethanol + water at $25^{\circ} \mathrm{C} / /$ Trans. Faraday Soc. 1953. V. 49, 890-895.

[45] Königer A., Meier B., Köhler W. Measurement of Soret, diffusion and thermal diffusion coefficients of three binary organic benchmark mixtures and of ethanol-water mixtures using a beam deflection technique // Philos. Magazine. 2009. V. 89, N 10, 907-923.

[46] M. Larrañaga, M. M. Bou-Ali, I. Lizarraga, J. A. Madariaga, and C. Santamaría. Soret coefficients of the ternary mixture 1,2,3,4-tetrahydronaphthalene + isobutylbenzene $+\mathrm{n}$ dodecane. J. Chem. Phys., 2015. V. 143, 024202.

[47] P. Blanco, M. M. Bou-Ali, J. K. Platten, D. A. Mezquia, J. A. Madariaga, C. Santamaría. Thermodiffusion coefficients of binary and ternary hydrocarbon mixtures. J. Chem. Phys., 2010. V. 132, 114506.

[48] A. Königer, H. Wunderlich, W. Köhler. Measurements of thermal diffusion in ternary mixtures using a two-color optical beam deflection technique. J. Chem. Phys., 2010. V. $132,174506$.

[49] D. R. Lide. Handbook of Chemixtry and physics. CRC Press, 84th edition, 2003-2004.

[50] Nist Chemistry Webbook, 2011.

[51] V. Sorokin, V. Frantskevich, L. Yanovsky, D. Bakulin, N. Dubovkin, V. Kotova / Edited by Yanovsky. Energy-intensive fuels for aviation and rocket engines. FIZMATLIT publishing house, Moscow, 2009 (in Russian).

[52] I. I. Ryzhkov, V. M. Shevtsova. On the cross-diffusion and the Soret effect in multicomponent mixtures. Micrograv. Sci. Technol., 2009. V. 21, issue 1, 014102.

[53] I. I. Ryzhkov and S. V. Kozlova. Stationary and transient Soret separation in a binary mixture with a consolute critical point, European Physical Journal E, 2016. V. 39, N 130. 